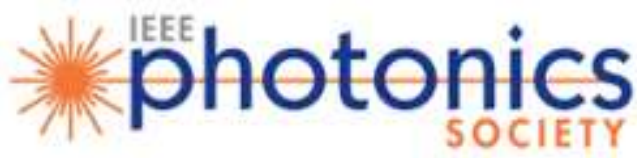

formerly $E$ EOS

\title{
Stabilization of dual-wavelength erbium doped ring fiber lasers by single-mode operation
}

\begin{tabular}{|c|c|}
\hline Journal: & Photonics Technology Letters \\
\hline Manuscript ID: & PTL-21123-2009.R1 \\
\hline Manuscript Type: & Original Paper \\
\hline \multicolumn{2}{|l|}{$\begin{array}{r}\text { Date Submitted by the } \\
\text { Author: }\end{array}$} \\
\hline Complete List of Authors: & $\begin{array}{l}\text { Quintela, Maria Angeles; University of Cantabria, Photonics } \\
\text { Engineering Group } \\
\text { Perez-Herrera, Rosa Ana; Public University of Navarra, Department } \\
\text { of Electric and Electronic Engineering } \\
\text { Canales, Irene; University of Cantabria, Photonics Engineering } \\
\text { Group } \\
\text { Fernández-Vallejo, Montserrat; Public University of Navarra, } \\
\text { Department of Electric and Electronic Engineering } \\
\text { Lopez-Amo, Manuel; Public University of Navarra, Department of } \\
\text { Electric and Electronic Engineering } \\
\text { Lopez-Higuera, Jose; Universidad de Cantabria, TEISA }\end{array}$ \\
\hline Key Words: & Erbium, Laser stability, Lasers, Gratings, Amplifiers \\
\hline
\end{tabular}

\section{(5) ScholaroNE




\title{
Stabilization of dual-wavelength erbium doped ring fiber lasers by single-mode operation
}

\author{
M. Angeles Quintela, Rosa Ana Pérez-Herrera, Irene Canales, Monserrat Fernández-Vallejo, Manuel \\ López-Amo Senior Member, IEEE and Jose Miguel Lopez-Higuera, Senior Member, IEEE
}

\begin{abstract}
In this work, a novel single-longitudinal-mode (SLM) dual-wavelength laser configuration is proposed and demonstrated. This laser is based on ring resonators, employs fiber Bragg gratings to select the operation wavelengths and includes a short piece of highly-doped Er-fibers acting as an active medium. The stable SLM operation is guaranteed when the two lasing channels present similar output powers. This behavior is shown for different pump powers.
\end{abstract}

Index Terms - Erbium-doped fiber (EDF), fiber Bragg grating (FBG), optical fiber ring laser, optical fiber amplifier, multiwavelength lasing.

\section{INTRODUCTION}

Stable multiwalength single-mode erbium doped fiber ring lasers (MEDFRL) are very attractive sources for many applications in optical fiber sensing, sensor network multiplexing schemes and instrument testing due to their advantages; simple structures, narrow linewidth, and compatibility with other optical fiber components [1], [2]. The selection of its operation wavelengths has been achieved by using different optical filtering techniques: Mach-Zehnder filter, arrayed waveguide gratings or fiber Bragg gratings (FBG) [3]-[5]. In addition to this, a variety of methods have been employed to try maximizing the number stable emission lines.

The erbium doped fiber ring lasers (EDFRL) usually generate multiple longitudinal modes around the central lasing wavelength due to its long cavity length and thus narrow longitudinal mode spacing. This aspect can limit their practical applications because of the mode competition and the mode hopping. To achieve single longitudinal mode (SLM) operation, several approaches have been proposed. In [6] a multi-ring cavity is proposed to guarantee a SLM operation. However, it is precise to adjust properly the laser parameters. In [7] a SLM operation of a fiber ring laser is achieved with a

Manuscrited received July 31, 2009. This work was supported in part by the Spanish Government project TEC2007-67987-C02 and the European project COST-299.

M. A. Q. I. C.and J. M. L. authors are with the Photonics Engineering Group, University of Cantabria, Laboratorio I+D Avda. Los Castros s/n, 39005 Spain (corresponding author to provide phone: +34942200879 ; fax: +34942200877; e-mail: quintelm@unican.es)

R.A. P.H., M. F. and M. L are with the Departmente of Electric and Electronic Engineering, Public University of Navarra, Pamplona, Spain. saturable absorber. Nevertheless, the efficiency of the fiber laser is reduced with this technique. A fiber Bragg grating Fabry-Perot etalon can be also used to obtain SLM fiber lasers, but the spacing between the lasing wavelengths can not be large [8].

The ring fiber lasers are also known to be susceptible to output power instabilities. These instabilities can degrade the performance characteristics of a sensor multiplexing network based on a laser interrogation scheme. The optimization of the ring laser configuration can improve considerably the characteristics of these lasers.

In this paper, a stable dual-wavelength erbium doped fiber ring laser that operates in SLM condition is experimentally proposed and demonstrated. The laser configuration is based on the serial connection of the FBG using optical circulators and the active medium is a highly-doped Er-fibers. This topology was previously reported by the authors in [9]-[11]. Experimental results of the time stability are also presented.

\section{EXPERIMENTAL SET UP}

The experimental setup of the proposed MEDFRL is shown in Fig. 1. This is a serial configuration based on circulators. The wavelength selection is carried out by means of FBGs. The FBGs are centered at $1543.6 \mathrm{nn}$ and $1550.9 \mathrm{~nm}$ with a corresponding full-width at half maximum (FWHM) of 0.842 $\mathrm{nm}$ and $0.61 \mathrm{~nm}$, respectively.

A highly erbium-doped fiber (EDF) (Er-30 by Liekki, with an absorption of $30 \mathrm{~dB} / \mathrm{m}$ at $\lambda=1530 \mathrm{~nm}$ ) is used, acting as the active medium. The length of this EDF was $7 \mathrm{~m}$. Because of the high concentration of erbium, the fiber length needed for the cavity was shorter than in other cases [10]. This configuration was also composed by a $1480 / 1550 \mathrm{~nm}$ wavelength division multiplexer (WDM), a $1480 \mathrm{~nm}$ pump source and a $3 \mathrm{~dB}$ coupler to incorporate the two FBGs into the laser cavity. To extract $2 \%$ of the laser output power from the ring, a $98 \%$ coupler is used.

In this configuration, two circulators were used to insert the FBGs reflected signals into the ring, ensuring unidirectional operation and therefore avoiding the spatial hole-burning (SHB) effect. Because of the use of circulators, inserting isolators were not necessary.

One of the major problems in multiwavelength ring lasers is that the oscillation threshold power for each wavelength is different due to the non-uniform shape of the EDF gain profile. As a consequence, variable attenuators (VA) have been 
connected to each FBG in order to correctly adjusting the cavity losses on each wavelength to achieve oscillation of the system in all the desired channels. All the free terminations on both systems have been immersed in refractive-index-matching gel to avoid undesired reflections.

\section{EXPERIMENTAL DEMONSTRATION}

The output spectrum of the MEDFRL for a $90 \mathrm{~mW}$ pump power is shown in Fig. 2. The experimental results of this work were obtained by using a high-resolution optical spectrum analyzer (BOSA-C Aragon Photonics) which measures optical spectra with a top combination of high resolution $(0.08 \mathrm{pm})$ and high dynamic range $(>80 \mathrm{~dB})$ at one time. As can be shown in Fig. 2, as there are two FBGs, two lasing channels could be obtained. The power of each of the two output channels is around $-16 \mathrm{dBm}$. For the two channels, the signal power is more than $45 \mathrm{~dB}$ higher than the amplified spontaneous emission (ASE) noise floor. The pumping threshold needed to obtain laser emission was around $45 \mathrm{~mW}$. However, we worked with higher pump power levels to increment the output power stability, as reported in [11].

The behavior of the longitudinal modes of this fiber laser was experimentally analyzed using this high-resolution optical spectrum analyzer. Its spectral resolution has a lower value than the mode spacing between the longitudinal modes of the ring, given by

$$
\Delta \lambda=\frac{\lambda^{2}}{n L}
$$

where $\mathrm{n}$ is the refractive index, $\mathrm{L}$ is the ring length and $\lambda$ is the central mode wavelength. As a consequence, we can verify the single longitudinal mode (SLM) operation condition.

We have measured different optical spectra corresponding to different values of pump powers and working conditions (dual or single-wavelength operations at $\lambda_{1}$ or $\lambda_{2}$ ), The optical spectrum of the first lasing channel $\left(\lambda_{1}=1543.6 \mathrm{~nm}\right)$ around centered mode wavelength in a single-wavelength operation for a pump power of $90 \mathrm{~mW}$ is shown by Fig. 3. As can be shown, multiple longitudinal modes are supported by the cavity. This spectral measurement was tested for several levels of pump power. We observed a broadening of the laser linewidth when the pump power increases. For example, for a pump power of $75 \mathrm{~mW}$, a FWHM linewidth of $3.25 \mathrm{GHz}$ was measured, however when a pump power of $90 \mathrm{~mW}$ was used, the FWHM linewidth increases to $4.5 \mathrm{GHz}$ The broadening of the laser linewidth is related with the irruption of additional longitudinal modes when the gain value rises up. Fig. 4 shows the output spectra of the two lasing channels in a dualwavelength operation for a reduced wavelength span around each centered mode wavelength. In this case, the pump power is $90 \mathrm{~mW}$ and the power difference between both channels is about $0.5 \mathrm{~dB}$. The optical spectrum of the first channel $\left(\lambda_{1}=\right.$ $1543.6 \mathrm{~nm}$ ) for a wavelength span of $56.4 \mathrm{pm}$ with 5.64 $\mathrm{pm} / \mathrm{div}$ is shown in Fig. 4(a). The one of the second channel $\left(\lambda_{2}=1550.9 \mathrm{~nm}\right)$ for a wavelength span of $47 \mathrm{pm}$ with 4,7 $\mathrm{pm} /$ div are shown in Fig 4(b).
As can be shown in these figures, this laser presents a SLM operation condition in both channels. These measurements have been repeated at different pump powers from $45 \mathrm{~mW}$ to $100 \mathrm{~mW}$. In all cases, a SLM operation in both channels is achieved when the two lasing wavelengths are oscillating simultaneously with similar output powers. The reason of this SLM operation when we have two channels oscillating at the same time is being studied currently and will be published elsewhere. A possible explanation of this behavior is the self injection seeding. The seed light of one channel is then produced from the other channel, and vice versa [12]. So that, the mode competition is reduced and an improved SLM operation in both channels is obtained.

The power of the laser output can suffer some changes with the time. For this reason, the temporal variations of the output power were also measured and analyzed on this MEDFRL. The instability is defined as the output power for a given interval of time (hours) and a specific confidence level (CL). This is the probability value associated with a confidence interval, given as a percentage. The confidence interval (CI) is the estimated range of values where the parameter of interest is included. We have tested the laser during a period of 6 hours. The measured data have been stored each 15 seconds and a CL of $90 \%$ was considered. Just to give a data, at room temperature the power averaged variation with $\mathrm{CL}=90 \%$ and Ppump $=100 \mathrm{~mW}$ was about $1.2 \mathrm{~dB}$ for $\lambda_{1}=1543.6 \mathrm{~nm}$, and about $0.9 \mathrm{~dB}$ for $\lambda_{2}=1550.9 \mathrm{~nm}$. Thus, we can conclude that both channels present good output power stability.

\section{CONCLUSION}

A stable dual-wavelength erbium-doped fiber ring laser has been achieved by using FBGs to perform wavelength selection on the systems. In a single-wavelength operation of this laser, we have experimentally demonstrated that multiple longitudinal modes are supported by the cavity. However, for similar pumping levels, we achieve a single-mode operation of the laser when we emit simultaneously two wavelengths using a special ring cavity configuration. The stable SLM operation is guaranteed if the output power of both channels is similar. This implies that it is possible to avoid the utilization of additional optical filtering techniques (that reduce the optical efficiency) to achieve the SLM operation. There is also good power stability of this laser that uses a serial topology involving circulators.

\section{ACKNOWLEDGMENT}

The authors want to thank Liekkis' fibers for having supplied the erbium doped fiber used in the experiments

\section{REFERENCES}

[1] A. Bellemare, "Continous-wave silica-based erbium-doped fibre lasers" Progress in Quantum Electron., vol. 27, no. 4, pp. 211-266, 2003.

[2] J.M. López-Higuera, "Handbook of Optical Fiber Sensing Technology", Wiley \& Sons, 2002.

[3] T. Miyazaki, N. Edagawa, S. Yamamoto, and S. Akiba, "A multiwavelength fiber ring-laser employing a pair of silica-based 
arrayed-waveguide-gratings," IEEE Photonics Technology Letters, vol. 9, n. 7, (1997), pp. 910-912.

[4] A. D. Kersey and W. W. Morey, "Multi-element Bragg-grating based Fiber-laser Strain Sensor," Electronics Letters, vol. 29, n. 11, (1993), pp. $964-966$.

[5] E. Achaerandio, S. Jarabo, S. Abad, and M. López-Amo, "New WDM Amplified Network for Optical Sensor Multiplexing," IEEE Photonics Technology Letters, vol. 11, n. 12, (1999), pp. 1644-1646.

[6] S. Pan; X., Zhao; C. Lou, "Switchable single-longitudinal-mode dualwavelength erbium-doped fiber ring laser incorporating a semiconductor optical amplifier," Optics Letters, vol. 33, n 8, (2008), pp 764-766.

[7] K. Zhang, J. Kang, "C-band wavelength-swept single-longitudinal-mode erbium-doped fiber ring laser," Optics Express, vol. 16, n 18, (2008), pp 14173-14179.

[8] J.L Zhou, L. Xia, X.P. Cheng, X.P. Dong, P. Shum, "Photonic generation of tunable microwave signals by beating a dual-wavelength single longitudinal mode fiber ring laser," Applied Physics B: Lasers and Optics, vol. 91, n 1, (2008), pp 99-103.

[9] R. A. Pérez-Herrera, M. Fernández, M. López-Amo, M. A. Quintela, A. Ullán and J. M. López-Higuera, "Comparison of ring resonador structures for multiwavelength fibre lasers using highly doped Erfibres", in Proc. 1st Mediterranean Photon. Conf., Ischia, Italy, 2008, pp. 88-90.

[10] L. Talaverano, S. Abad, S. Jarabo, and M. López-Amo, "Multiwavelength Fiber Laser Sources with Bragg-Grating Sensor Multiplexing Capability," Journal of Lightwave Technology, vol. 19, n. 4, (2001), pp. 553-558.

[11] M. A. Quintela, A. Quintela, J. M. Lopez-Higuera, R. A. Pérez-Herrera, M. Fernández and M. López-Amo, "Output power and wavelength stability experimental study of two fibre laser structures", in Proc. 1st Mediterranean Photon. Conf., Ischia, Italy, 2008, pp. 140-142.

[12] P.D. Dragic, "Analytical model for injection-seeded erbium-doped fiber ring lasers," IEEE Photonics Technology Letters, vol. 17, n 8, (2005), pp 1629-1631.

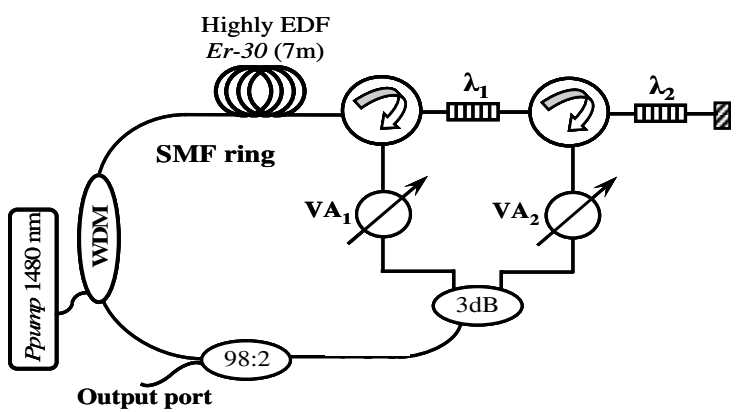

Fig.1. Experimental setup of the EDFRL using a serial topology involving circulators.

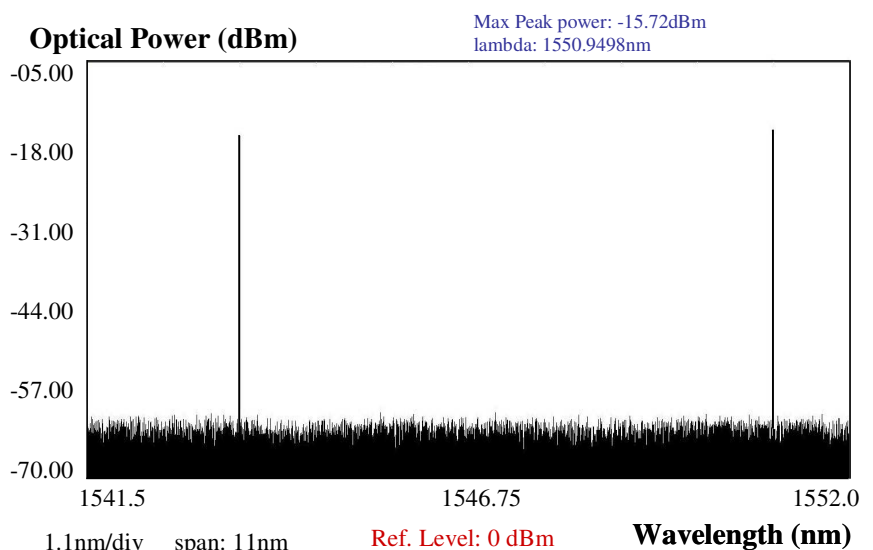

Fig. 2. Output optical spectrum measured by the BOSA for the MEDFRL circulators configuration and a pump power of $90 \mathrm{~mW}$.

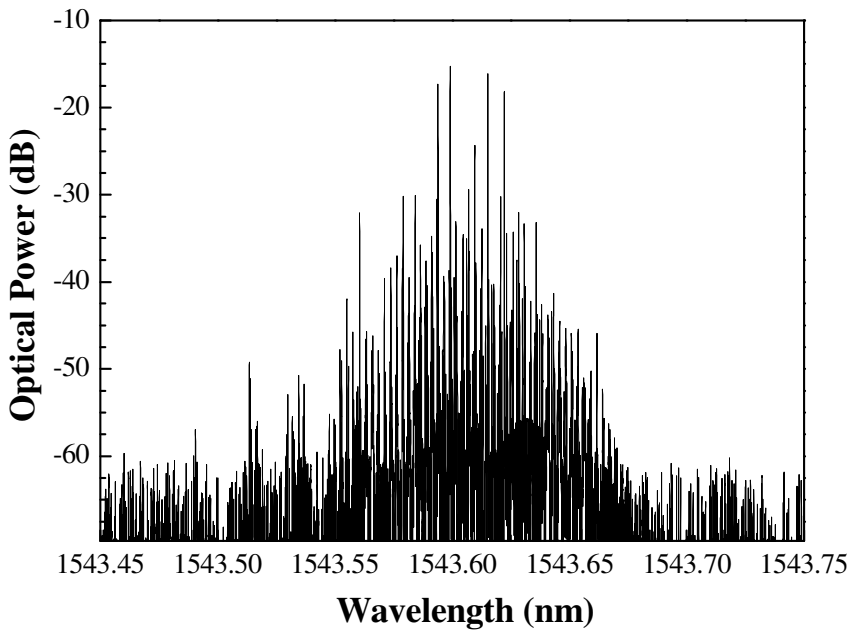

Fig. 3. Detail of the output optical spectrum for the first channel $\left(\lambda_{1}=1543.6\right.$ $\mathrm{nm})$ at single-wavelength operation.

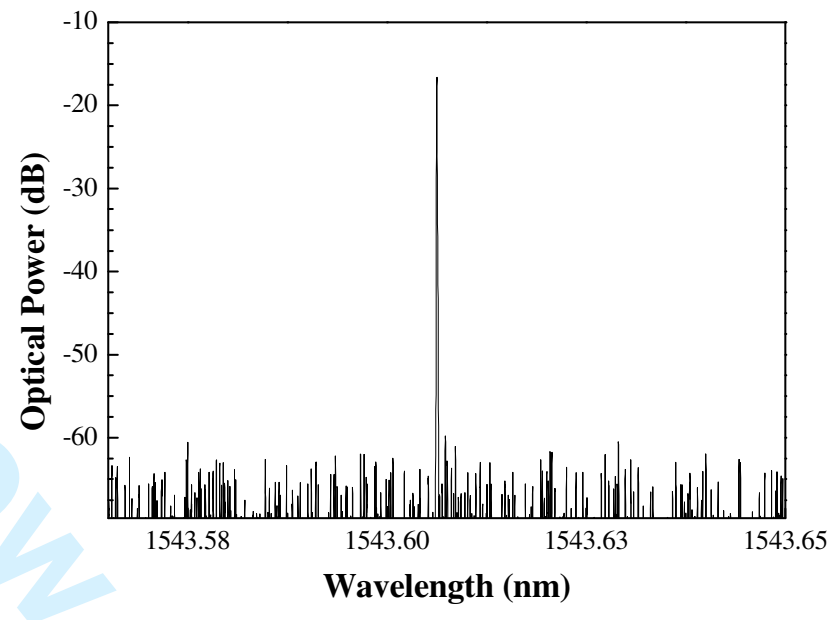

(a)

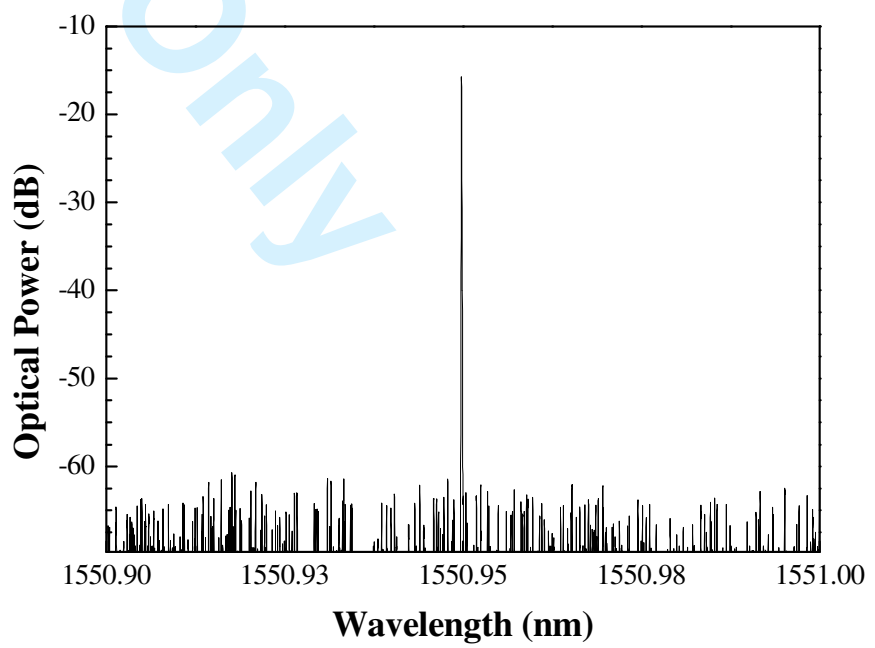

(b)

Fig. 4. Details of the output optical spectrum (a) for the first channel $\left(\lambda_{1}=\right.$ $1543.6 \mathrm{~nm})$ and $(b)$ for the second channel $\left(\lambda_{1}=1550.9 \mathrm{~nm}\right)$ at dualwavelength operation. 


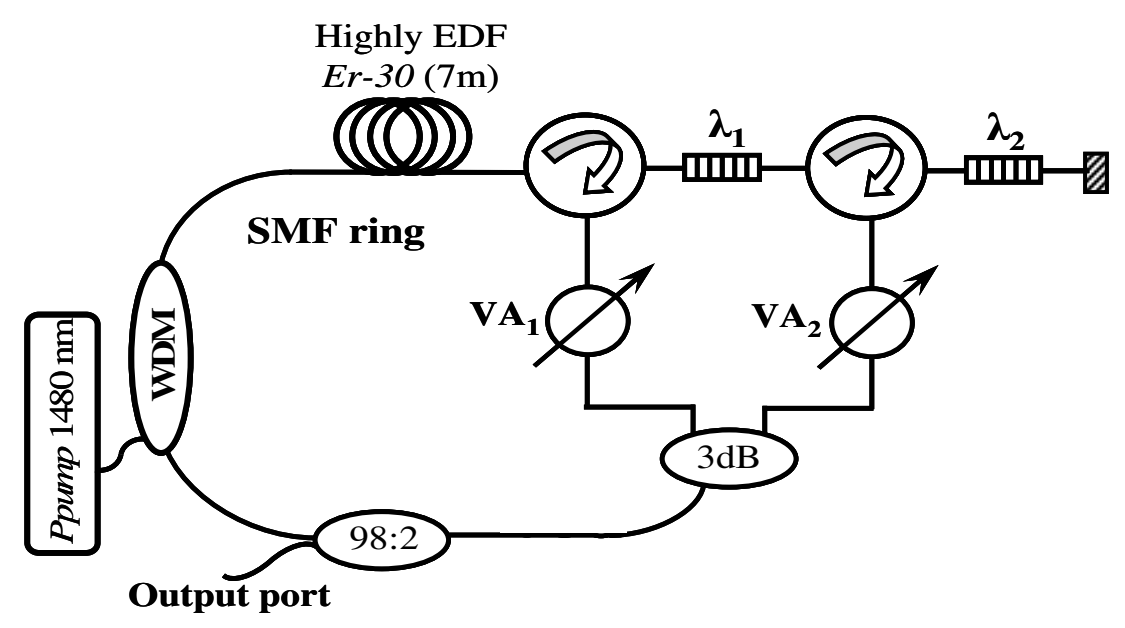




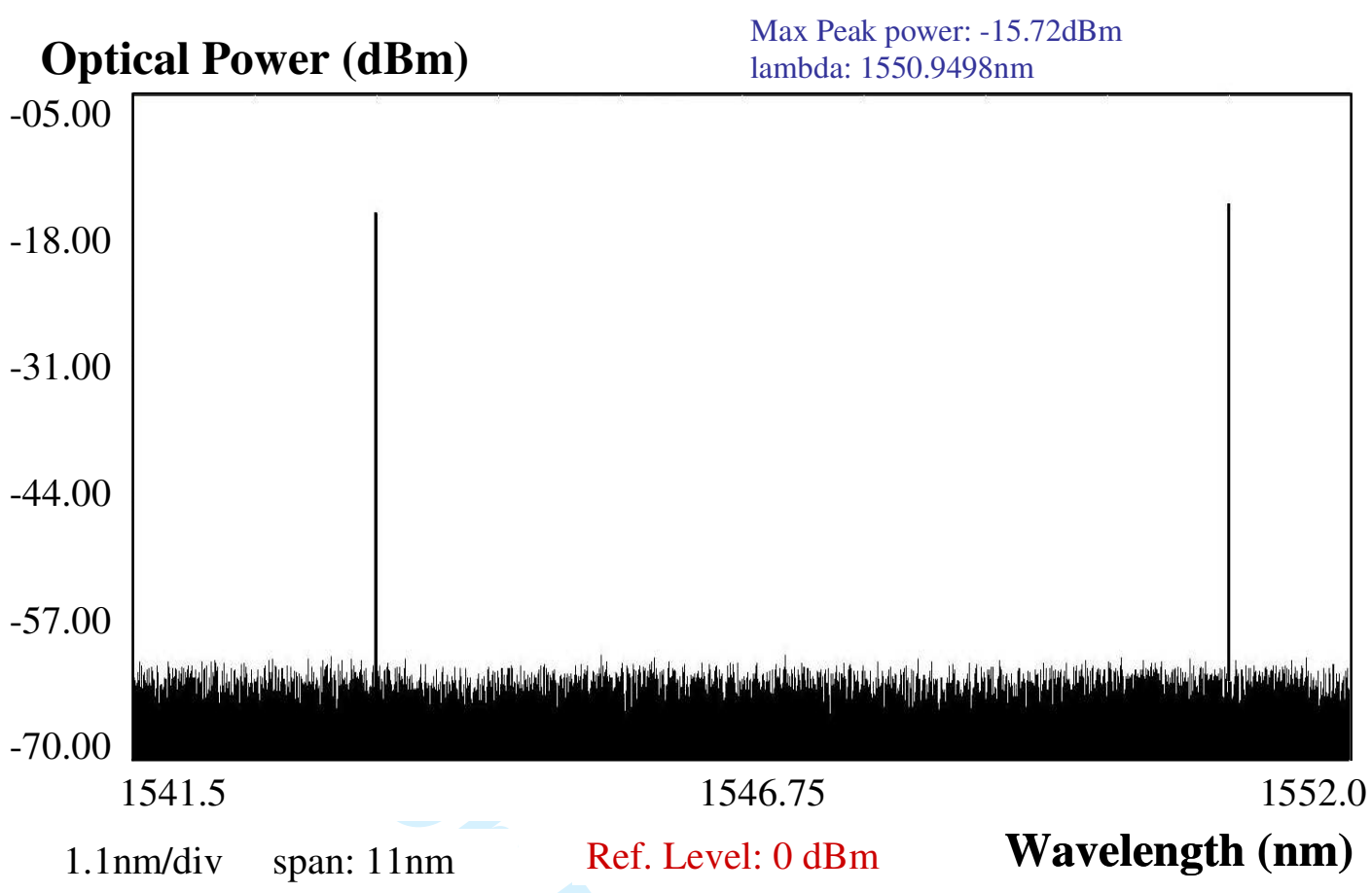




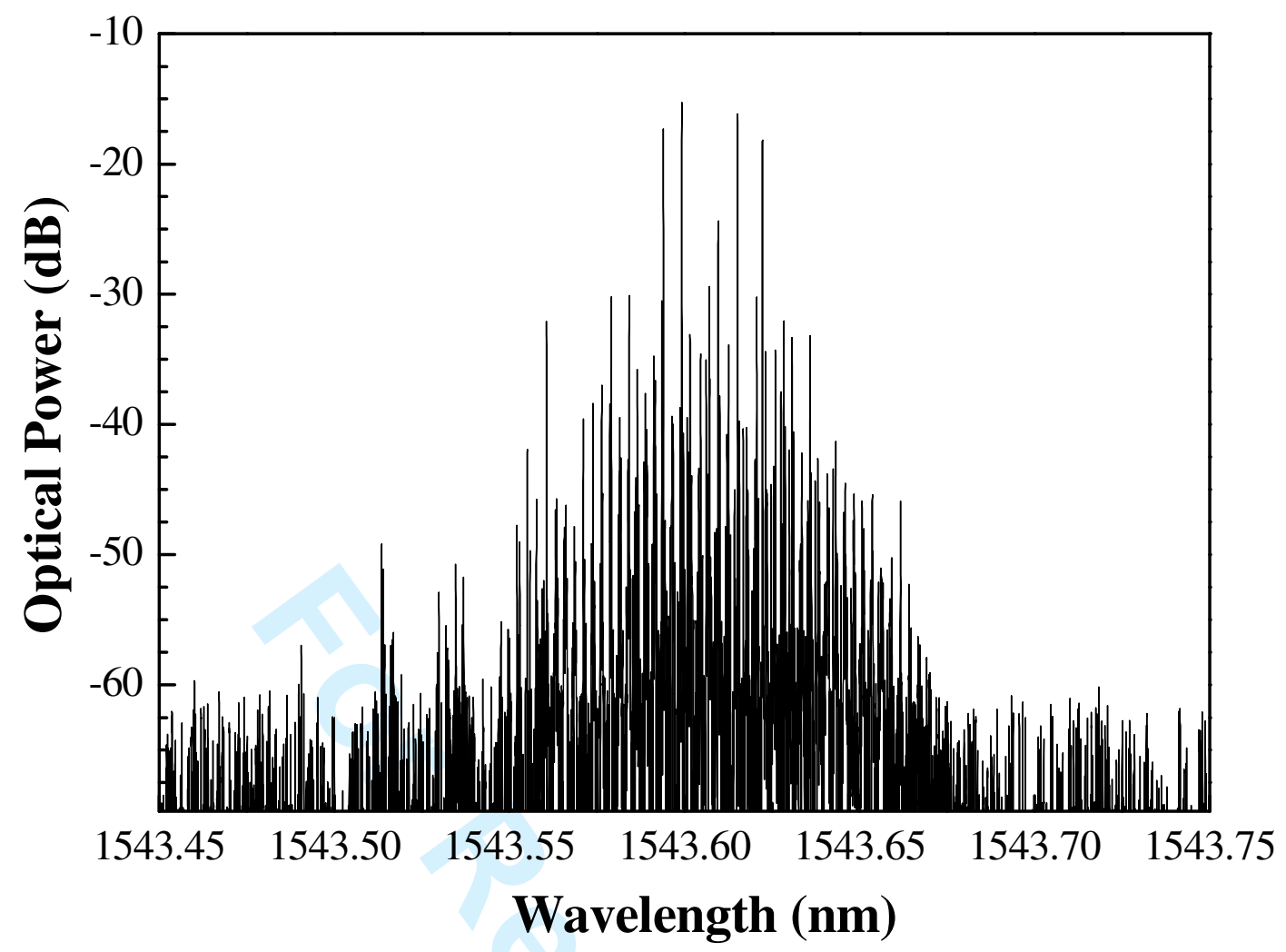




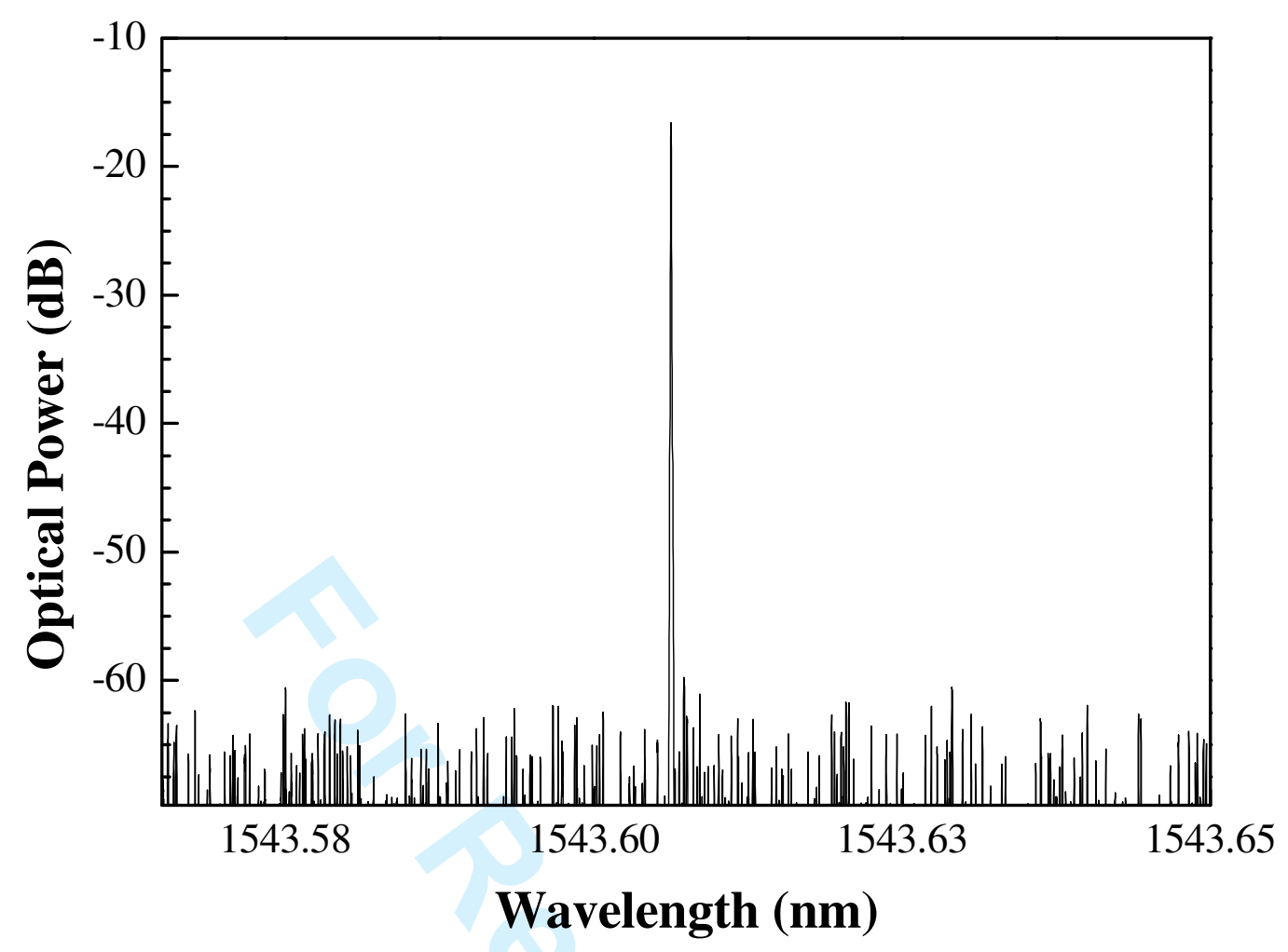




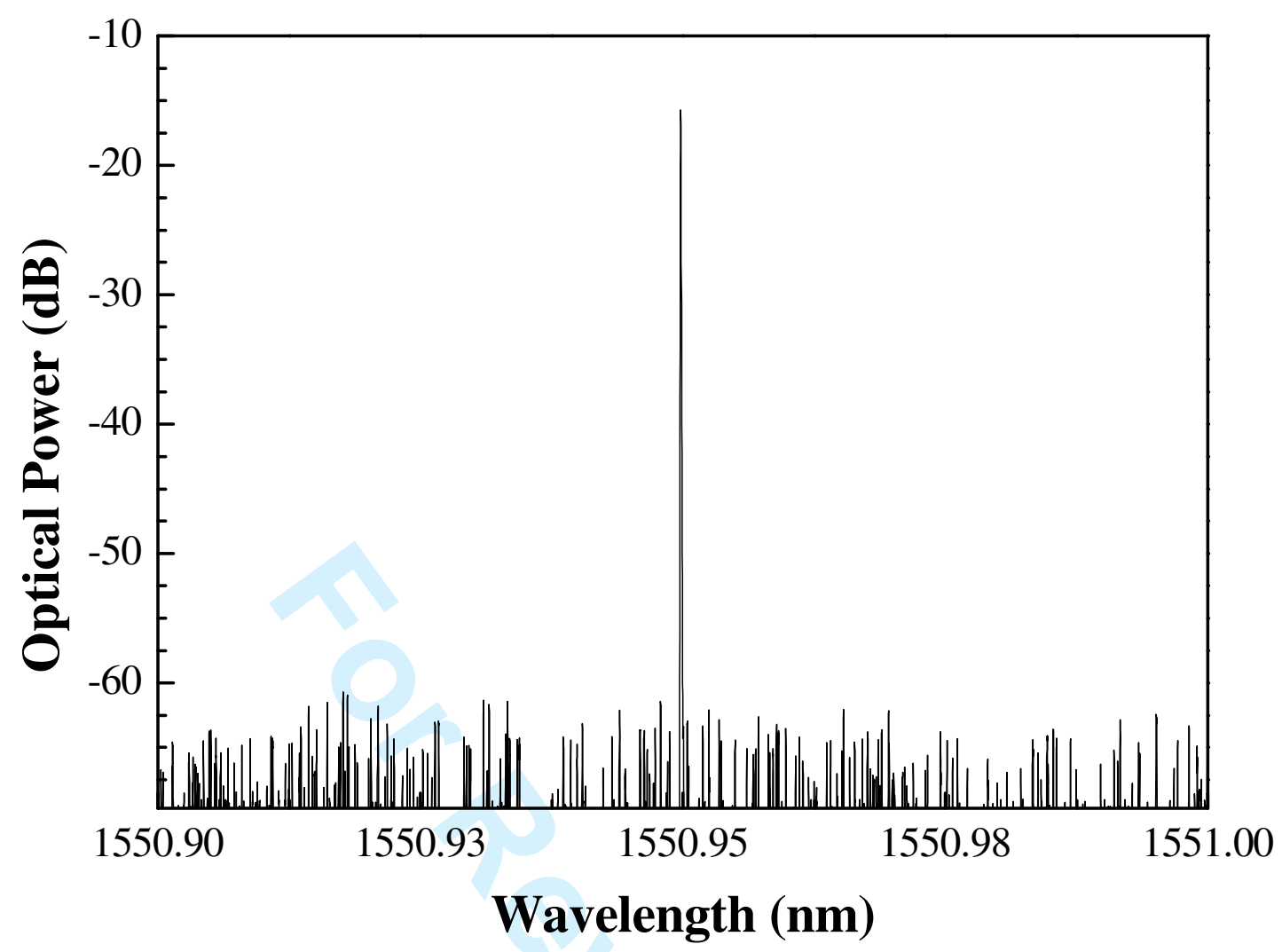




\section{Reviewer: 1}

We appreciate the positive referee's comments. We agree with all remarks and comments. And we have taken them into consideration in the revised manuscript in order to improve its quality.

In the following pages each of the referee demands are answered and changes in the paper highlighted (see Version with changes.pdf file).

1. The paper tried to demonstrate a single-longitudinal-mode (SLM) dual-wavelength ring laser, but the demonstration was not convincing. As point out in [R1], two issues must be carefully addressed to ensure a stable operation of an SLM dual-wavelength erbium-doped fiber laser. The first is to reduce the mode competition and the second is to eliminate multi-longitudinal-mode oscillation.

In order to clarify these aspects next paragraph has been included in order to explain that once the SLM has been obtained the mode competition and mode hopping is not produced:

As reported in previous works [12] a SLM fiber ring laser can be made to annihilate the mode competition with an auxiliary lasing. In this work, owing to the interaction of the seed light produced from one channel to the other one and vice versa [13], multiplelongitudinal-mode oscillation can be suppressed, as can be seen in Fig 4(a) and (b), and thus the mode competition and mode hopping is not produced. Therefore, the laser oscillation is rather stable.

2. For the first issue, the authors refer to self injection seeding (but no details are provided). To my best knowledge, the dual-wavelength operation in this configuration is due to carefully balancing of the cavity loss of the two wavelength channels [R2] and is also because of the relative large wavelength spacing $(7.3 \mathrm{~nm})$. The operation of such lasers has been well and sufficiently studied.

In fact, in order to obtain a simultaneous dual-wavelength operation in this configuration, firstly we select two FBGs inside the amplification band of the Er-doped fiber to generate the lasing wavelengths and afterwards the cavity losses of each wavelength have to be adjusted; in our case using variable attenuators (VAs). This explanation can be found in the next paragraph of the paper:

One of the major problems in multiwavelength ring lasers is that the oscillation threshold power for each wavelength is different due to the non-uniform shape of the EDF gain profile. As a consequence, variable attenuators (VA) have been connected to each FBG in order to correctly adjusting the cavity losses on each wavelength to achieve oscillation of the system in all the desired channels.

3. For the second issue, the authors at first gave Fig. 3 to show that the laser easily oscillates with multiple longitudinal modes, and then released Fig. 4 to describe the SLM oscillation (no explain is provided to address the conversion from multiple modes to single mode), which is totally confusing. 
Once a simultaneous dual-wavelength operation is obtained, next step consists of annihilating the multi-longitudinal-mode oscillation of each channel that is, obtaining monomode behavior for each channel $\left(\lambda_{1}\right.$ and $\left.\lambda_{2}\right)$. In this work a SLM operation in both channels has been achieved when the two lasing wavelengths are oscillating simultaneously with similar output powers by using the VAs to adjust the cavity losses.

The reason of this SLM operation when we have two channels oscillating at the same time is being studied currently and will be published elsewhere. A SML operation can be also obtained by using an external source instead of the one of the FBGs or even by means of different wavelength spacing between the FBGs, as we experimentally carried out in a number of preliminary studies. In addition to this, that SML operation has been verified for different pump power levels. Because of this, we can conclude, such as is shown in the paper, that:

In all cases (for all the studied pump power levels), a SLM operation in both channels is achieved when the two lasing wavelengths are oscillating simultaneously with similar output powers by using the VAs to adjust the cavity losses. [...]

This SML operation can be also obtained by using an external source instead of the one of the FBGs or even by means of different wavelength spacing between the FBGs, as we experimentally carried out in a number of preliminary studies. [...]

In this work, owing to the interaction of the seed light produced from one channel to the other one and vice versa [13], multiple-longitudinal-mode oscillation can be suppressed, as can be seen in Fig 4(a) and (b), and thus the mode competition and mode hopping is not produced. Therefore, the laser oscillation is rather stable.

On the other hand, Fig 3 shows the optical spectrum for the first channel $\left(\lambda_{1}\right)$ when only this channel is oscillating. This situation was obtained using the VAs to adjust the cavity losses. In that case, multiple-longitudinal-modes appear around the central wavelength $\left(\lambda_{1}\right)$, as can be seen in this figure.

However Fig 4 shows the optical spectrum when both channels are simultaneously oscillating with a very low power difference between the two channels (around $0.5 \mathrm{~dB}$ ). Under these work conditions a SML operation is obtained for both channels at the same time.

In order to clarify this aspect this paragraph has been modified as next:

Fig. 4 shows the output spectra of the two lasing channels in a dual-wavelength operation with similar output powers for a reduced wavelength span around each centered mode wavelength.

4. In addition, the laser has a similar configuration as that reported in [9]-[11], so the paper lacks novelty.

As the reviewer suggested this configuration has been previously reported in [9]-[11] by the authors. However, in these previous works an excellent temporal stability was experimentally demonstrated against typical configurations but the longitudinal mode for different laser conditions were either analyzed or studied. Due to this is an important aspect for the different applications of the multiwavelength lasers. 
1

3

4

5

6

7

8

9

10

11

12

13

In this work has been experimentally demonstrated that, thanks to the advantages of the innovative BOSA-C technology, and by using that configuration, a SLM operation can be obtained for some particular system requirements.

In order to highlight this novelty, next paragraph has been added at the end of the first section.

However, in this paper, the possibility to obtain a single-mode behavior is experimentally demonstrated by using a high-resolution optical spectrum analyzer. Experimental results of the good time stability obtained in the single-mode regime are also presented. 


\section{Reviewer: 2}

We appreciate the positive referee's comments. We agree with all remarks and comments. And we have taken them into consideration in the revised manuscript in order to improve its quality.

In the following pages each of the referee demands are answered and changes in the paper highlighted (see Version with changes.pdf file).

Recommendation: $\mathrm{C}=$ This Paper Is Borderline. The Recommendation To Publish Or Reject Depends On The Authors Response To My Comments.

Comments:

This paper experimentally demonstrates a dual-wavelength erbium doped fiber ring laser which operates in single longitudinal mode (SLM). Such laser configuration with circulators was previously proposed by the authors (J. LIGHTW. TECHNOL. Vol. 27 No. 14. pp.2563) and showed some advantages such as good stability both in emission power and wavelength. Here, the authors report their further discovery that SLM operation can also be obtained in such topology as long as the output power from two channels is similar. The experimental results are interesting and valuable for SLM research area. In conclusion, the manuscript could be suitable for publication if the authors can address the following points.

1. The reflectivity of two FBG should be added.

We totally agree with the referee so this information has been added to the paper.

The wavelength selection is carried out by means of FBGs with a reflectivity of about $98 \%$.

2. It is well known that mode hopping is inevitable for fiber ring lasers with long cavity. The author's comment leaves the impression that the laser could always operate in stable SLM and mode-hopping never happens. The comment on mode-hopping of the laser should be added.

As the reviewer suggested, this new paragraph and one more reference about modehopping has been included:

As reported in previous works [12] a SLM fiber ring laser can be made to annihilate the mode competition with an auxiliary lasing. In this work, owing to the interaction of the seed light produced from one channel to the other one and vice versa [13], multiplelongitudinal-mode oscillation can be suppressed, as can be seen in Fig 4(a) and (b), and thus the mode competition and mode hopping is not produced. Therefore, the laser oscillation is rather stable.

[12] J. Sun and L. Huang, "Single-longitudinal-mode fiber ring laser using internal lasing injection and self-injection feedback," Optical Engineering, vol. 46, n. 7, (2007), pp. 074201-1-6. 
3. The authors measured a broad bandwidth when in a single-wavelength operation. Could the authors add the linewidth value in dual-wavelength operation?

In order to answer this question, this sentence has been included.

Also for this pump power level, a FWHM linewidth of $7.5 \mathrm{MHz}$ and $6 \mathrm{MHz}$ were measured for the first and second channels in that order.

4. Can the laser sustain SLM operation if the pump power is much larger than $90 \mathrm{~mW}$ ? The SLM operation can only be achieved in a limited range?

Yes, this laser can sustain SLM operation if the pump power is higher than $90 \mathrm{~mW}$. Besides, the SLM operation is not limited for a specific pump power range. These measurements were repeated at different pump powers from $45 \mathrm{~mW}$ to $100 \mathrm{~mW}$ due to the available source we used only works in this range, so we chose a pump power of $90 \mathrm{~mW}$ only as a sample of this behavior.

In addition to this, as it was previously proposed by the authors [11], using this configuration, a good stability both in emission power and wavelength were obtained from a pump power of about $90 \mathrm{~mW}$.

In order to clarify these aspects next paragraphs have been included:

These measurements have been repeated at different pump powers from $45 \mathrm{~mW}$ to 100 $m W$. In all cases, a SLM operation in both channels is achieved when the two lasing wavelengths are oscillating simultaneously with similar output powers by using the VAs to adjust the cavity losses. As it was previously proposed by the authors [11], using this configuration, a good stability both in emission power and wavelength were obtained from a pump power of about $90 \mathrm{~mW}$.

Also reference [11] has been replaced by next one in order to clear up this feature:

[11] R.A. Pérez-Herrera, M.A. Quintela, M. Fernández-Vallejo, A. Quintela, M. López-Amo, J.M. López-Higuera, "Stability comparison of two ring resonator structures for multiwavelength fiber lasers using highly doped Er-fibers," Journal of Lightwave Technology, vol. 27, n. 14, (2009), pp. 2563-2569.

5. The authors attribute the single longitudinal mode operation to self injection seeding. This explanation is too simple and hard to understand. Some more detailed analysis is expected.

Regarding to this and as the reviewer previously suggested, this new paragraph and one more reference about mode-hopping has been included:

As reported in previous works [12] a SLM fiber ring laser can be made to annihilate the mode competition with an auxiliary lasing. In this work, owing to the interaction of the seed light produced from one channel to the other one and vice versa [13], multiplelongitudinal-mode oscillation can be suppressed, as can be seen in Fig 4(a) and (b), 
and thus the mode competition and mode hopping is not produced. Therefore, the laser oscillation is rather stable.

[12] J. Sun and L. Huang, "Single-longitudinal-mode fiber ring laser using internal lasing injection and self-injection feedback," Optical Engineering, vol. 46, n. 7, (2007), pp. 074201-1-6. 


\section{Reviewer: 3}

We appreciate the positive referee's comments. We agree with all remarks and comments. And we have taken them into consideration in the revised manuscript in order to improve its quality.

In the following page each of the referee demands are answered and changes in the paper highlighted (please see Version with changes.pdf file).

Recommendation: $\mathrm{B}=$ This Paper Contains New And Important Information But Requires Moderate Revisions.

Comments:

The authors present a simple cavity configuration to realize a stable, dual-wavelngth, single longitudinal mode operation at around $1550 \mathrm{~nm}$. The paper is well written. However there are a few technical details (listed below) that I believe would require for it to become a publishable article.

1. Since there is no polarization controlling elements inside the ring cavities - did the author notice any change in laser stability while adjusting the VAs for different levels of pump powers? What limits the maximum pump power of $100 \mathrm{~mW}$ ?

When similar output power was obtained for both channels (by properly using the VAs), any change in laser stability was notice for different levels of pump powers. In order to clarify this question, next paragraph has been added to the paper. The pump power limitation comes from our available pump lasers.

In all cases, a SLM operation in both channels is achieved when the two lasing wavelengths are oscillating simultaneously with similar output powers by using the VAs to adjust the cavity losses.

2. Page 2, col. 1, line 17 - "For the two channels .... (ASE) noise floor". Does this statement hold true for low OSA resolution settings i.e. $1 \mathrm{~nm}$ ?

The high-resolution optical spectrum analyzer used was a BOSA-C, which directly measures the peak power levels. Therefore, the values obtained are independent of the spectral resolution used, unlike the values measured with an OSA where the resulting measurement is related to the spectral power density.

3. Page 2, col. 1, line 27 - "Its spectral resolution .... (SLM) operation condition". The authors mentioned the length of the EDF only but there is no indicaiton of the total cavity length of the two rings to perform the necessary calculations.

We totally agree with the referee comment so this information has been included in the text:

The length of this EDF was $7 \mathrm{~m}$ and the total cavity length was $14 \mathrm{~m}$.

4. Page 2, col. 2, line 10 - "A possible explanation ... both channels is obtained." According to reference 12, one would require substantial amount of seed power for 
injection locking a laser. Can the authors explain the origin of such large seed powers assuming FBGs have relatively high sideband suppression $(\sim 20 \mathrm{~dB})$ ?

The SML operation can be also obtained by using an external source instead of the one of the FBGs, as we experimentally carried out in parallel studies. Because of this, we can conclude that the sideband suppression of the FBGs it is not relevant in our case to obtain the seed power for injection locking the laser, being the reflectivity of the FBGs the key parameter.

5. The authors claimed that the source is temporally stable and provided some numbers to justify the claim. The source might be stable on a longer time scale but I would like to see the rf plots (electrical spectra as shown in reference 6) of the two competing wavelengths showing both low and high frequency components (from $\mathrm{Hz}$ to hundreds of $\mathrm{MHz}$ ) to support the stability claim. The current Figure 4 which is simply zooming the spectra of the two wavelengths already captured in Figure 2 can be removed to accommodate these rf plots.

This work has been totally carried out in the optical domain due to the advantages of the innovative BOSA-C technology, unlike previous works about this aim where the use of electrical spectrum analyzer was essential in order to study the SLM operation (such as is shown in [6]), with the corresponding drawbacks. This BOSA technology is the reunion of real high resolution with high dynamic range at a time and for a real time measurement. 


\title{
Stabilization of dual-wavelength erbium doped ring fiber lasers by single-mode operation
}

\author{
M. Angeles Quintela, Rosa Ana Pérez-Herrera, Irene Canales, Monserrat Fernández-Vallejo, Manuel \\ López-Amo Senior Member, IEEE and Jose Miguel Lopez-Higuera, Senior Member, IEEE
}

\begin{abstract}
In this work, a novel single-longitudinal-mode (SLM) dual-wavelength laser configuration is proposed and demonstrated. This laser is based on ring resonators, and employs fiber Bragg gratings to select the operation wavelengths. It includes a short piece of highly-doped Er-fiber that acts as the active medium. The stable SLM operation is guaranteed when the two lasing channels present similar output powers. This behavior is shown for different pump powers.
\end{abstract}

Index Terms - Erbium-doped fiber (EDF), fiber Bragg grating (FBG), optical fiber ring laser, optical fiber amplifier, multiwavelength lasing.

\section{INTRODUCTION}

Stable multiwalength single-mode erbium doped fiber ring lasers (MEDFRL) are very attractive sources for many applications in optical fiber sensing, sensor network multiplexing schemes and instrument testing due to their advantages; simple structures, narrow linewidth, and compatibility with other optical fiber components [1], [2]. The selection of its operation wavelengths has been achieved by using different optical filtering techniques: Mach-Zehnder filter, arrayed waveguide gratings or fiber Bragg gratings (FBG) [3]-[5]. In addition to this, a variety of methods have been employed to try maximizing the number stable emission lines.

The erbium doped fiber ring lasers (EDFRL) usually generate multiple longitudinal modes around the central lasing wavelength due to its long cavity length and thus narrow longitudinal mode spacing. This aspect can limit their practical applications because of the mode competition and the mode hopping. To achieve single longitudinal mode (SLM) operation, several approaches have been proposed [6]-[8]. In [6] a multi-ring cavity is proposed to guarantee a SLM operation. However, it is precise to adjust properly the laser parameters. In [7] a SLM operation of a fiber ring laser is

Manuscrited received July 31, 2009. This work was supported in part by the Spanish Government project TEC2007-67987-C02 and the European project COST-299.

M. A. Q. I. C.and J. M. L. authors are with the Photonics Engineering Group, University of Cantabria, Laboratorio I+D Avda. Los Castros $\mathrm{s} / \mathrm{n}$, 39005 Spain (corresponding author to provide phone: +34942200879 ; fax: +34942200877; e-mail: quintelm@unican.es)

R.A. P.H., M. F. and M. L-A are with the Department of Electric and Electronic Engineering, Public University of Navarra, Pamplona, Spain. achieved with a saturable absorber. Nevertheless, the efficiency of the fiber laser is reduced with this technique. A fiber Bragg grating Fabry Perot etalon can be also used to obtain SLM fiber lasers, but the spacing between the lasing wavelengths can not be large [8].

The ring fiber lasers are also known to be susceptible to output power instabilities. These instabilities can degrade the performance characteristics of a sensor multiplexing network based on a laser interrogation scheme. The optimization of the ring laser configuration can improve considerably the characteristics of these lasers.

In this paper, a stable dual-wavelength erbium doped fiber ring laser that operates in SLM condition is experimentally proposed and demonstrated. The laser configuration is based on the serial connection of the FBG using optical circulators and the active medium is a highly-doped Er-fibers. This topology was previously reported by the authors in [9]-[11]. However, in this paper, the possibility to obtain a single-mode behavior is experimentally demonstrated by using a highresolution optical spectrum analyzer. Experimental results of the good time stability obtained in the single-mode regime are also presented.

\section{EXPERIMENTAL SET UP}

The experimental setup of the proposed MEDFRL is shown in Fig. 1. This is a serial configuration based on circulators. The wavelength selection is carried out by means of FBGs with a reflectivity of about $98 \%$. These FBGs are centered at $1543.6 \mathrm{nn}$ and $1550.9 \mathrm{~nm}$ with a corresponding full-width at half maximum (FWHM) of $0.842 \mathrm{~nm}$ and $0.61 \mathrm{~nm}$, respectively.

A highly erbium-doped fiber (EDF) (Er-30 by Liekki, with an absorption of $30 \mathrm{~dB} / \mathrm{m}$ at $\lambda=1530 \mathrm{~nm}$ ) is used, acting as the active medium. The length of this EDF was $7 \mathrm{~m}$ and the total cavity length was $14 \mathrm{~m}$. Because of the high concentration of erbium, the fiber length needed for the cavity was shorter than in other cases [10]. This configuration was also composed by a 1480/1550nm wavelength division multiplexer (WDM), a $1480 \mathrm{~nm}$ pump source and a $3 \mathrm{~dB}$ coupler to incorporate the two FBGs into the laser cavity. To extract $2 \%$ of the laser output power from the ring, a $98 \%$ coupler is used.

In this configuration, two circulators were used to insert the FBGs reflected signals into the ring, ensuring unidirectional operation and therefore avoiding the spatial hole-burning (SHB) effect. Because of the use of circulators, it was not 
necessary to introduce isolators inside the cavity.

One of the major problems in multiwavelength ring lasers is that the oscillation threshold power for each wavelength is different due to the non-uniform shape of the EDF gain profile. As a consequence, variable attenuators (VA) have been connected to each FBG in order to correctly adjusting the cavity losses on each wavelength to achieve oscillation of the system in all the desired channels. All the free terminations on both systems have been immersed in refractive-index-matching gel to avoid undesired reflections.

\section{EXPERIMENTAL DEMONSTRATION}

The output spectrum of the MEDFRL for a $90 \mathrm{~mW}$ pump power is shown in Fig. 2. The experimental results of this work were obtained by using a high-resolution optical spectrum analyzer (BOSA-C Aragon Photonics) which measures the eptical spectrum that offers simultaneously a with a top combination of high resolution $(0.08 \mathrm{pm})$ and a high dynamic range $(>80 \mathrm{~dB})$ at one time. As can be shown in Fig. 2, as there are using two FBGs, two lasing channels eould be are obtained. The power of each of the two output channels is around $-16 \mathrm{dBm}$. For the two channels, the signal power is more than $45 \mathrm{~dB}$ higher than the amplified spontaneous emission (ASE) noise floor. The pumping threshold needed to obtain laser emission was around $45 \mathrm{~mW}$. However, we worked with higher pump power levels to increment the output power stability, as reported in [11].

The behavior of the longitudinal modes of this fiber laser was experimentally analyzed using this high-resolution optical spectrum analyzer. Its spectral resolution has a lower value than the mode spacing between the longitudinal modes of the ring, given by

$$
\Delta \lambda=\frac{\lambda^{2}}{n L}
$$

where $\mathrm{n}$ is the refractive index, $\mathrm{L}$ is the ring length and $\lambda$ is the central mode wavelength. As a consequence, we can verify the single longitudinal mode (SLM) operation condition.

We have measured different optical spectra corresponding to different values of pump powers and working conditions (dual or single-wavelength operations at $\lambda_{1}$ or $\lambda_{2}$ ). The optical spectrum of the first lasing channel $\left(\lambda_{1}=1543.6 \mathrm{~nm}\right)$ around centered mode wavelength in a single-wavelength operation for a pump power of $90 \mathrm{~mW}$ is shown by Fig. 3. As can be shown, multiple longitudinal modes are supported by the cavity. This spectral measurement was tested for several levels of pump power. We observed a broadening of the laser linewidth when the pump power increases. For example, for a pump power of $75 \mathrm{~mW}$, a FWHM linewidth of $3.25 \mathrm{GHz}$ was measured, however when a pump power of $90 \mathrm{~mW}$ was used, the FWHM linewidth increases to $4.5 \mathrm{GHz}$. The broadening of the laser linewidth is related with the irruption of additional longitudinal modes when the gain value rises. Fig. 4 shows the output spectra of the two lasing channels in a dual-wavelength operation with similar output powers for a reduced wavelength span around each centered single-mode emitted wavelength. In this case, the pump power is $90 \mathrm{~mW}$ and the power difference between both channels is about $0.5 \mathrm{~dB}$. The optical spectrum of the first channel $\left(\lambda_{1}=1543.6 \mathrm{~nm}\right)$ for a wavelength span of $56.4 \mathrm{pm}$ with $5.64 \mathrm{pm} / \mathrm{div}$ is shown in Fig. 4(a). The one of the second channel $\left(\lambda_{2}=1550.9 \mathrm{~nm}\right)$ for a wavelength span of 47 pm with $4.7 \mathrm{pm} / \mathrm{div}$ are shown in Fig 4(b). Also for this pump power level, a FWHM linewidth of $7.5 \mathrm{MHz}$ and $6 \mathrm{MHz}$ were measured for the first and second channels in that order.

As can be shown in these figures, this laser presents a SLM operation condition in both channels. These measurements have been repeated at different pump powers from $45 \mathrm{~mW}$ to $100 \mathrm{~mW}$. In all cases, a SLM operation in both channels is achieved when the two lasing wavelengths are oscillating simultaneously with similar output powers by using the VAs to adjust the cavity losses. As it was previously proposed by the authors [11], using this configuration, a good stability both in emission power and wavelength were obtained from a pump power of about $90 \mathrm{~mW}$.

This SML operation can be also obtained by using an external source instead of the one of the FBGs or even by means of different wavelength spacing between the FBGs, as we experimentally carried out in a number of preliminary studies. The reason of this SLM operation when we have two channels oscillating at the same time is being studied currently and will be published elsewhere. A possible explanation of this behavior is the self injection seeding. The seed light of one channel is then produced from the other channel, and vice versa [13]. So that, the mode competition is reduced and an improved SLM operation in both channels is obtained. As reported in previous works [12] a SLM fiber ring laser can be made to annihilate the mode competition with an auxiliary lasing. In this work, owing to the interaction of the seed light produced from one channel to the other one and vice versa [13], multiple-longitudinal-mode oscillation can be suppressed, as can be seen in Fig 4(a) and (b), and thus the mode competition and mode hopping is not produced. Therefore, the laser oscillation is rather stable.

The power of the laser output can suffer some changes with the time. For this reason, the temporal variations of the output power were also measured and analyzed on this MEDFRL. The instability is defined as the output power for a given interval of time (hours) and a specific confidence level (CL). This is the probability value associated with a confidence interval, given as a percentage. The confidence interval (CI) is the estimated range of values where the parameter of interest is included. We have tested the laser during a period of 6 hours. The measured data have been stored each 15 seconds and a CL of $90 \%$ was considered. Just to give a data, at room temperature the power averaged variation with $\mathrm{CL}=90 \%$ and Ppump $=100 \mathrm{~mW}$ was about $1.2 \mathrm{~dB}$ for $\lambda_{1}=1543.6 \mathrm{~nm}$, and about $0.9 \mathrm{~dB}$ for $\lambda_{2}=1550.9 \mathrm{~nm}$. Thus, we can conclude that both channels present good output power stability.

\section{CONCLUSION}

A stable dual-wavelength erbium-doped fiber ring laser has been achieved by using FBGs to perform wavelength selection 
on the systems. In a single-wavelength operation of this laser, we have experimentally demonstrated that multiple longitudinal modes are supported by the cavity. However, for similar pumping levels, we achieve a single-mode operation of the laser when we emit simultaneously two wavelengths using a special ring cavity configuration. The stable SLM operation is guaranteed if the output power of both channels is similar. This implies that it is possible to avoid the utilization of additional optical filtering techniques (that reduce the optical efficiency) to achieve the SLM operation. There is also good power stability of this laser that uses a serial topology involving circulators.

\section{ACKNOWLEDGMENT}

The authors want to thank Liekkis' fibers for having supplied the erbium doped fiber used in the experiments and to Santigo Tainta for his help in the edition of the manuscript.

\section{REFERENCES}

[1] A. Bellemare, "Continous-wave silica-based erbium-doped fibre lasers" Progress in Quantum Electron., vol. 27, no. 4, pp. 211-266, 2003.

[2] J.M. López-Higuera, "Handbook of Optical Fiber Sensing Technology", Wiley \& Sons, 2002.

[3] T. Miyazaki, N. Edagawa, S. Yamamoto, and S. Akiba, "A multiwavelength fiber ring-laser employing a pair of silica-based arrayed-waveguide-gratings," IEEE Photonics Technology Letters, vol. 9, n. 7, (1997), pp. 910-912.

[4] A. D. Kersey and W. W. Morey, "Multi-element Bragg-grating based Fiber-laser Strain Sensor," Electronics Letters, vol. 29, n. 11, (1993), pp. $964-966$.

[5] E. Achaerandio, S. Jarabo, S. Abad, and M. López-Amo, "New WDM Amplified Network for Optical Sensor Multiplexing," IEEE Photonics Technology Letters, vol. 11, n. 12, (1999), pp. 1644-1646.

[6] S. Pan; X., Zhao; C. Lou, "Switchable single-longitudinal-mode dualwavelength erbium-doped fiber ring laser incorporating a semiconductor optical amplifier," Optics Letters, vol. 33, n 8, (2008), pp 764-766.

[7] K. Zhang, J. Kang, "C-band wavelength-swept single-longitudinal-mode erbium-doped fiber ring laser," Optics Express, vol. 16, n 18, (2008), pp 14173-14179.

[8] J.L Zhou, L. Xia, X.P. Cheng, X.P. Dong, P. Shum, "Photonic generation of tunable microwave signals by beating a dual-wavelength single longitudinal mode fiber ring laser," Applied Physics B: Lasers and Optics, vol. 91, n 1, (2008), pp 99-103.

[9] R. A. Pérez-Herrera, M. Fernández, M. López-Amo, M. A. Quintela, A. Ullán and J. M. López-Higuera, "Comparison of ring resonador structures for multiwavelength fibre lasers using highly doped Erfibres", in Proc. 1st Mediterranean Photon. Conf., Ischia, Italy, 2008, pp. 88-90.

[10] L. Talaverano, S. Abad, S. Jarabo, and M. López-Amo, "Multiwavelength Fiber Laser Sources with Bragg-Grating Sensor Multiplexing Capability," Journal of Lightwave Technology, vol. 19, n. 4, (2001), pp. 553-558.

[11] R.A. Pérez-Herrera, M.A. Quintela, M. Fernández-Vallejo, A. Quintela, M. López-Amo, J.M. López-Higuera, "Stability comparison of two ring resonator structures for multiwavelength fiber lasers using highly doped Er-fibers," Journal of Lightwave Technology, vol. 27, n. 14, (2009), pp. 2563-2569.

[12] J. Sun and L. Huang, "Single-longitudinal-mode fiber ring laser using internal lasing injection and self-injection feedback," Optical Engineering, vol. 46, n. 7, (2007), pp. 074201-1-6.

[13] P.D. Dragic, "Analytical model for injection-seeded erbium-doped fiber ring lasers," IEEE Photonics Technology Letters, vol. 17, n 8, (2005), pp 1629-1631.

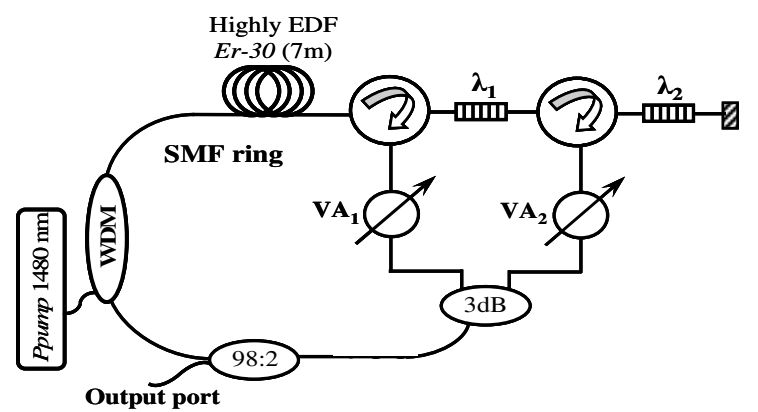

Fig.1. Experimental setup of the EDFRL using a serial topology involving circulators.

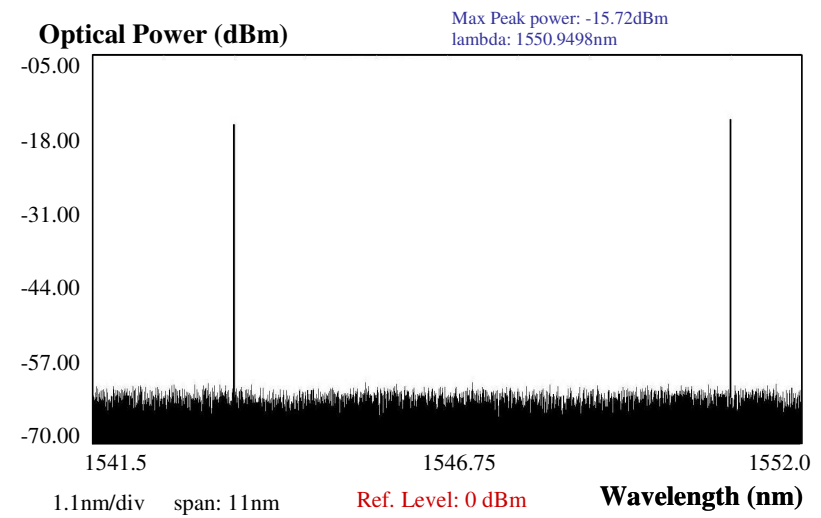

Fig. 2. Output optical spectrum measured by the BOSA for the MEDFRL circulators configuration and a pump power of $90 \mathrm{~mW}$.

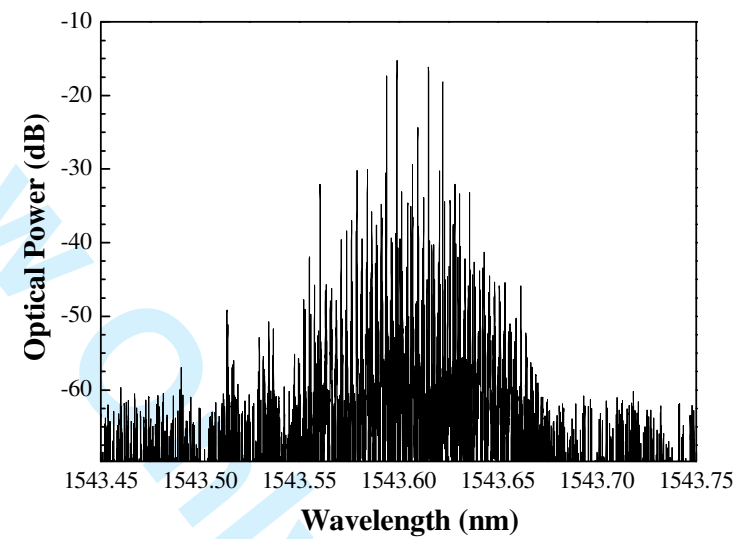

Fig. 3. Detail of the output optical spectrum for the first channel $\left(\lambda_{1}=1543.6\right.$ $\mathrm{nm})$ at single-wavelength operation.

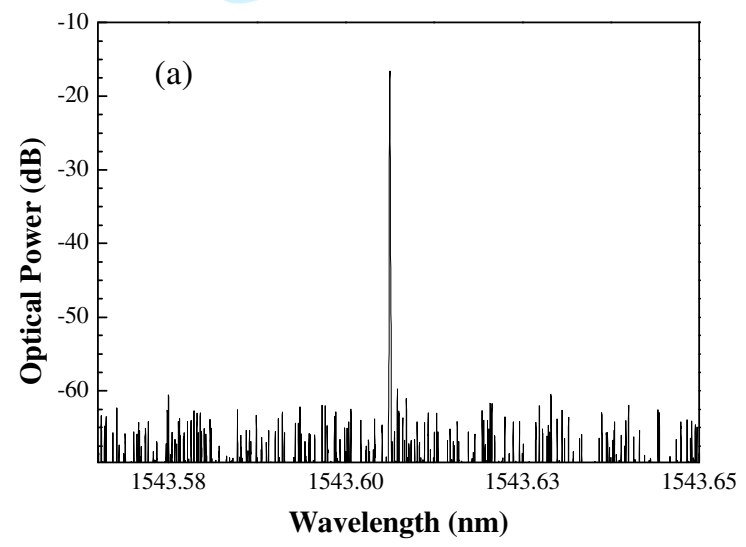




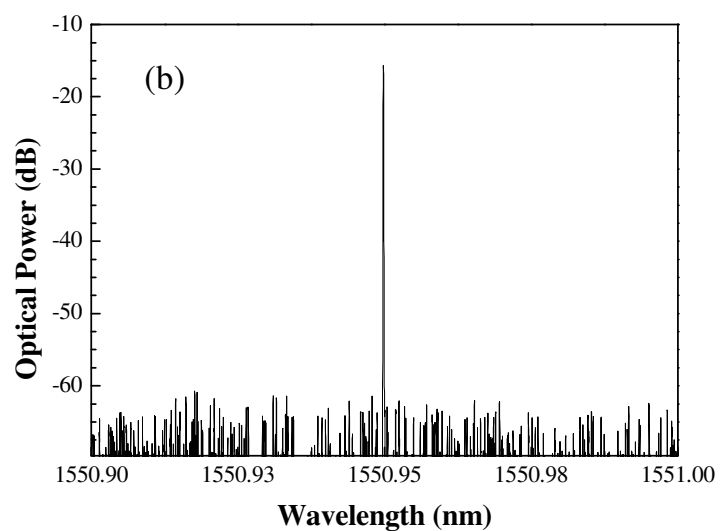

Fig. 4. Details of the output optical spectrum (a) for the first channel $\left(\lambda_{1}=\right.$ $1543.6 \mathrm{~nm})$ and (b) for the second channel $\left(\lambda_{1}=1550.9 \mathrm{~nm}\right)$ at dualwavelength operation. 


\title{
Stabilization of dual-wavelength erbium doped ring fiber lasers by single-mode operation
}

\author{
M. Angeles Quintela, Rosa Ana Pérez-Herrera, Irene Canales, Monserrat Fernández-Vallejo, Manuel \\ López-Amo Senior Member, IEEE and Jose Miguel Lopez-Higuera, Senior Member, IEEE
}

\begin{abstract}
In this work, a novel single-longitudinal-mode (SLM) dual-wavelength laser configuration is proposed and demonstrated. This laser is based on ring resonators, and employs fiber Bragg gratings to select the operation wavelengths. It includes a short piece of highly-doped Er-fiber that acts as the active medium. The stable SLM operation is guaranteed when the two lasing channels present similar output powers. This behavior is shown for different pump powers.
\end{abstract}

Index Terms - Erbium-doped fiber (EDF), fiber Bragg grating (FBG), optical fiber ring laser, optical fiber amplifier, multiwavelength lasing.

\section{INTRODUCTION}

Stable multiwalength single-mode erbium doped fiber ring lasers (MEDFRL) are very attractive sources for many applications in optical fiber sensing, sensor network multiplexing schemes and instrument testing due to their advantages; simple structures, narrow linewidth, and compatibility with other optical fiber components [1], [2]. The selection of its operation wavelengths has been achieved by using different optical filtering techniques: Mach-Zehnder filter, arrayed waveguide gratings or fiber Bragg gratings (FBG) [3]-[5]. In addition to this, a variety of methods have been employed to try maximizing the number stable emission lines.

The erbium doped fiber ring lasers (EDFRL) usually generate multiple longitudinal modes around the central lasing wavelength due to its long cavity length. This aspect can limit their practical applications because of the mode competition and the mode hopping. To achieve single longitudinal mode (SLM) operation, several approaches have been proposed [6][8]. The ring fiber lasers are also known to be susceptible to output power instabilities. These instabilities can degrade the performance characteristics of a sensor multiplexing network based on a laser interrogation scheme. The optimization of the ring laser configuration can improve considerably the

Manuscrited received July 31, 2009. This work was supported in part by the Spanish Government project TEC2007-67987-C02 and the European project COST-299.

M. A. Q. I. C.and J. M. L. authors are with the Photonics Engineering Group, University of Cantabria, Laboratorio I+D Avda. Los Castros s/n, 39005 Spain (corresponding author to provide phone: +34942200879 ; fax: +34942200877; e-mail: quintelm@unican.es)

R.A. P.H., M. F. and M. L-A are with the Department of Electric and Electronic Engineering, Public University of Navarra, Pamplona, Spain. characteristics of these lasers.

In this paper, a stable dual-wavelength erbium doped fiber ring laser that operates in SLM condition is experimentally proposed and demonstrated. The laser configuration is based on the serial connection of the FBG using optical circulators and the active medium is a highly-doped Er-fibers. This topology was previously reported by the authors in [9]-[11]. However, in this paper, the possibility to obtain a single-mode behavior is experimentally demonstrated by using a highresolution optical spectrum analyzer. Experimental results of the good time stability obtained in the single-mode regime are also presented.

\section{EXPERIMENTAL SET UP}

The experimental setup of the proposed MEDFRL is shown in Fig. 1. This is a serial configuration based on circulators. The wavelength selection is carried out by means of FBGs with a reflectivity of about $98 \%$. These FBGs are centered at $1543.6 \mathrm{nn}$ and $1550.9 \mathrm{~nm}$ with a corresponding full-width at half maximum (FWHM) of $0.842 \mathrm{~nm}$ and $0.61 \mathrm{~nm}$, respectively.

A highly erbium-doped fiber (EDF) (Er-30 by Liekki, with an absorption of $30 \mathrm{~dB} / \mathrm{m}$ at $\lambda=1530 \mathrm{~nm}$ ) is used, acting as the active medium. The length of this EDF was $7 \mathrm{~m}$ and the total cavity length was $14 \mathrm{~m}$. Because of the high concentration of erbium, the fiber length needed for the cavity was shorter than in other cases [10]. This configuration was also composed by a 1480/1550nm wavelength division multiplexer (WDM), a $1480 \mathrm{~nm}$ pump source and a $3 \mathrm{~dB}$ coupler to incorporate the two FBGs into the laser cavity. To extract $2 \%$ of the laser output power from the ring, a 98\% coupler is used.

In this configuration, two circulators were used to insert the FBGs reflected signals into the ring, ensuring unidirectional operation and therefore avoiding the spatial hole-burning (SHB) effect. Because of the use of circulators, it was not necessary to introduce isolators inside the cavity.

One of the major problems in multiwavelength ring lasers is that the oscillation threshold power for each wavelength is different due to the non-uniform shape of the EDF gain profile. As a consequence, variable attenuators (VA) have been connected to each FBG in order to correctly adjusting the cavity losses on each wavelength to achieve oscillation of the system in all the desired channels. All the free terminations on both systems have been immersed in refractive-indexmatching gel to avoid undesired reflections. 


\section{EXPERIMENTAL DEMONSTRATION}

The output spectrum of the MEDFRL for a $90 \mathrm{~mW}$ pump power is shown in Fig. 2. The experimental results of this work were obtained by using a high-resolution optical spectrum analyzer (BOSA-C Aragon Photonics) which offers simultaneously a high resolution $(0.08 \mathrm{pm})$ and a high dynamic range $(>80 \mathrm{~dB})$. As can be shown in Fig. 2, using two FBGs, two lasing channels are obtained. The power of each of the two output channels is around $-16 \mathrm{dBm}$. For the two channels, the signal power is more than $45 \mathrm{~dB}$ higher than the amplified spontaneous emission (ASE) noise floor. The pumping threshold needed to obtain laser emission was around $45 \mathrm{~mW}$. However, we worked with higher pump power levels to increment the output power stability, as reported in [11].

The behavior of the longitudinal modes of this fiber laser was experimentally analyzed using this high-resolution optical spectrum analyzer. Its spectral resolution has a lower value than the mode spacing between the longitudinal modes of the ring, given by

$$
\Delta \lambda=\frac{\lambda^{2}}{n L}
$$

where $n$ is the refractive index, $L$ is the ring length and $\lambda$ is the central mode wavelength. As a consequence, we can verify the single longitudinal mode (SLM) operation condition.

We have measured different optical spectra corresponding to different values of pump powers and working conditions (dual or single-wavelength operations at $\lambda_{1}$ or $\lambda_{2}$ ). The optical spectrum of the first lasing channel $\left(\lambda_{1}=1543.6 \mathrm{~nm}\right)$ around centered mode wavelength in a single-wavelength operation for a pump power of $90 \mathrm{~mW}$ is shown by Fig. 3. As can be shown, multiple longitudinal modes are supported by the cavity. This spectral measurement was tested for several levels of pump power. We observed a broadening of the laser linewidth when the pump power increases. For example, for a pump power of $75 \mathrm{~mW}$, a FWHM linewidth of $3.25 \mathrm{GHz}$ was measured, however when a pump power of $90 \mathrm{~mW}$ was used, the FWHM linewidth increases to $4.5 \mathrm{GHz}$. The broadening of the laser linewidth is related with the irruption of additional longitudinal modes when the gain value rises. Fig. 4 shows the output spectra of the two lasing channels in a dual-wavelength operation with similar output powers for a reduced wavelength span around each single-mode emitted wavelength. In this case, the pump power is $90 \mathrm{~mW}$ and the power difference between both channels is about $0.5 \mathrm{~dB}$. The optical spectrum of the first channel $\left(\lambda_{1}=1543.6 \mathrm{~nm}\right)$ for a wavelength span of $56.4 \mathrm{pm}$ with $5.64 \mathrm{pm} / \mathrm{div}$ is shown in Fig. 4(a). The one of the second channel $\left(\lambda_{2}=1550.9 \mathrm{~nm}\right)$ for a wavelength span of $47 \mathrm{pm}$ with $4.7 \mathrm{pm} / \mathrm{div}$ are shown in Fig 4(b). Also for this pump power level, a FWHM linewidth of 7.5 $\mathrm{MHz}$ and $6 \mathrm{MHz}$ were measured for the first and second channels in that order.

As can be shown in these figures, this laser presents a SLM operation condition in both channels. These measurements have been repeated at different pump powers from $45 \mathrm{~mW}$ to $100 \mathrm{~mW}$. In all cases, a SLM operation in both channels is achieved when the two lasing wavelengths are oscillating simultaneously with similar output powers by using the VAs to adjust the cavity losses. As it was previously proposed by the authors [11], using this configuration, a good stability both in emission power and wavelength were obtained from a pump power of about $90 \mathrm{~mW}$.

This SML operation can be also obtained by using an external source instead of the one of the FBGs or even by means of different wavelength spacing between the FBGs, as we experimentally carried out in a number of preliminary studies. The reason of this SLM operation when we have two channels oscillating at the same time is being studied currently and will be published elsewhere. A possible explanation of this behavior is the self injection seeding.

As reported in previous works [12] a SLM fiber ring laser can be made to annihilate the mode competition with an auxiliary lasing. In this work, owing to the interaction of the seed light produced from one channel to the other one and vice versa [13], multiple-longitudinal-mode oscillation can be suppressed, as can be seen in Fig 4(a) and (b), and thus the mode competition and mode hopping is not produced. Therefore, the laser oscillation is rather stable.

The power of the laser output can suffer some changes with the time. For this reason, the temporal variations of the output power were also measured and analyzed on this MEDFRL. The instability is defined as the output power for a given interval of time (hours) and a specific confidence level (CL). This is the probability value associated with a confidence interval, given as a percentage. The confidence interval (CI) is the estimated range of values where the parameter of interest is included. We have tested the laser during a period of 6 hours. The measured data have been stored each 15 seconds and a CL of $90 \%$ was considered. Just to give a data, at room temperature the power averaged variation with $\mathrm{CL}=90 \%$ and Ppump $=100 \mathrm{~mW}$ was about $1.2 \mathrm{~dB}$ for $\lambda_{1}=1543.6 \mathrm{~nm}$, and about $0.9 \mathrm{~dB}$ for $\lambda_{2}=1550.9 \mathrm{~nm}$. Thus, we can conclude that both channels present good output power stability.

\section{CONCLUSION}

A stable dual-wavelength erbium-doped fiber ring laser has been achieved by using FBGs to perform wavelength selection on the systems. In a single-wavelength operation of this laser, we have experimentally demonstrated that multiple longitudinal modes are supported by the cavity. However, for similar pumping levels, we achieve a single-mode operation of the laser when we emit simultaneously two wavelengths using a special ring cavity configuration. The stable SLM operation is guaranteed if the output power of both channels is similar. This implies that it is possible to avoid the utilization of additional optical filtering techniques (that reduce the optical efficiency) to achieve the SLM operation. There is also good power stability of this laser that uses a serial topology involving circulators. 


\section{ACKNOWLEDGMENT}

The authors want to thank Liekkis' fibers for having supplied the erbium doped fiber used in the experiments and to Santigo Tainta for his help in the edition of the manuscript.

\section{REFERENCES}

[1] A. Bellemare, "Continous-wave silica-based erbium-doped fibre lasers" Progress in Quantum Electron., vol. 27, no. 4, pp. 211-266, 2003.

[2] J.M. López-Higuera, "Handbook of Optical Fiber Sensing Technology", Wiley \& Sons, 2002.

[3] T. Miyazaki, N. Edagawa, S. Yamamoto, and S. Akiba, "A multiwavelength fiber ring-laser employing a pair of silica-based arrayed-waveguide-gratings," IEEE Photonics Technology Letters, vol. 9, n. 7, (1997), pp. 910-912.

[4] A. D. Kersey and W. W. Morey, "Multi-element Bragg-grating based Fiber-laser Strain Sensor," Electronics Letters, vol. 29, n. 11, (1993), pp. 964-966.

[5] E. Achaerandio, S. Jarabo, S. Abad, and M. López-Amo, "New WDM Amplified Network for Optical Sensor Multiplexing," IEEE Photonics Technology Letters, vol. 11, n. 12, (1999), pp. 1644-1646.

[6] S. Pan; X., Zhao; C. Lou, "Switchable single-longitudinal-mode dualwavelength erbium-doped fiber ring laser incorporating a semiconductor optical amplifier," Optics Letters, vol. 33, n 8, (2008), pp 764-766.

[7] K. Zhang, J. Kang, "C-band wavelength-swept single-longitudinal-mode erbium-doped fiber ring laser," Optics Express, vol. 16, n 18, (2008), pp 14173-14179.

[8] J.L Zhou, L. Xia, X.P. Cheng, X.P. Dong, P. Shum, "Photonic generation of tunable microwave signals by beating a dual-wavelength single longitudinal mode fiber ring laser," Applied Physics B: Lasers and Optics, vol. 91, n 1, (2008), pp 99-103.

[9] R. A. Pérez-Herrera, M. Fernández, M. López-Amo, M. A. Quintela, A. Ullán and J. M. López-Higuera, "Comparison of ring resonador structures for multiwavelength fibre lasers using highly doped Erfibres", in Proc. 1st Mediterranean Photon. Conf., Ischia, Italy, 2008, pp. 88-90.

[10] L. Talaverano, S. Abad, S. Jarabo, and M. López-Amo, "Multiwavelength Fiber Laser Sources with Bragg-Grating Sensor Multiplexing Capability," Journal of Lightwave Technology, vol. 19, n. 4, (2001), pp. 553-558.

[11] R.A. Pérez-Herrera, M.A. Quintela, M. Fernández-Vallejo, A. Quintela, M. López-Amo, J.M. López-Higuera, "Stability comparison of two ring resonator structures for multiwavelength fiber lasers using highly doped Er-fibers," Journal of Lightwave Technology, vol. 27, n. 14, (2009), pp. 2563-2569.

[12] J. Sun and L. Huang, "Single-longitudinal-mode fiber ring laser using internal lasing injection and self-injection feedback," Optical Engineering, vol. 46, n. 7, (2007), pp. 074201-1-6.

[13] P.D. Dragic, "Analytical model for injection-seeded erbium-doped fiber ring lasers," IEEE Photonics Technology Letters, vol. 17, n 8, (2005), pp 1629-1631.

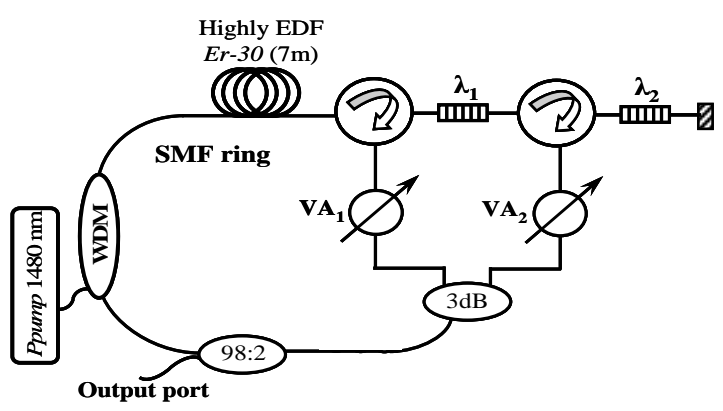

Fig.1. Experimental setup of the EDFRL using a serial topology involving circulators.

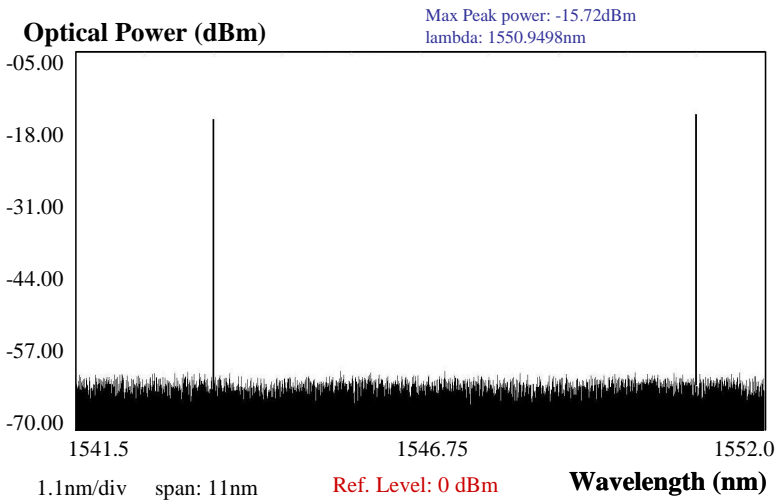

Fig. 2. Output optical spectrum measured by the BOSA for the MEDFRL circulators configuration and a pump power of $90 \mathrm{~mW}$.

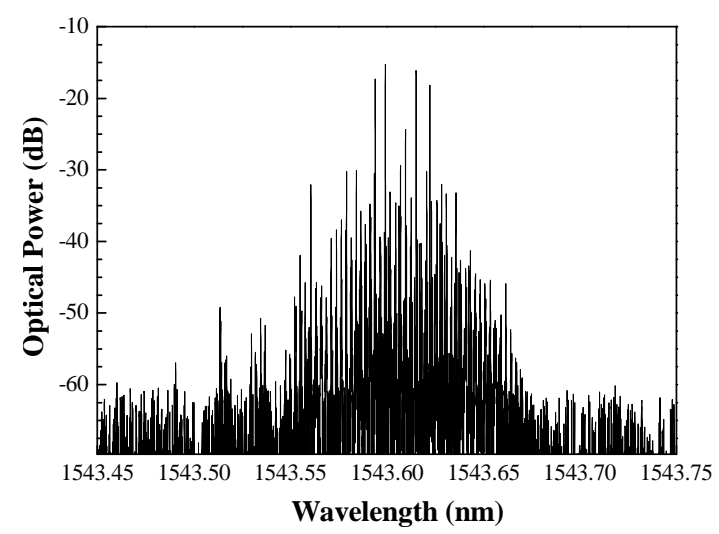

Fig. 3. Detail of the output optical spectrum for the first channel $\left(\lambda_{1}=1543.6\right.$ $\mathrm{nm})$ at single-wavelength operation.
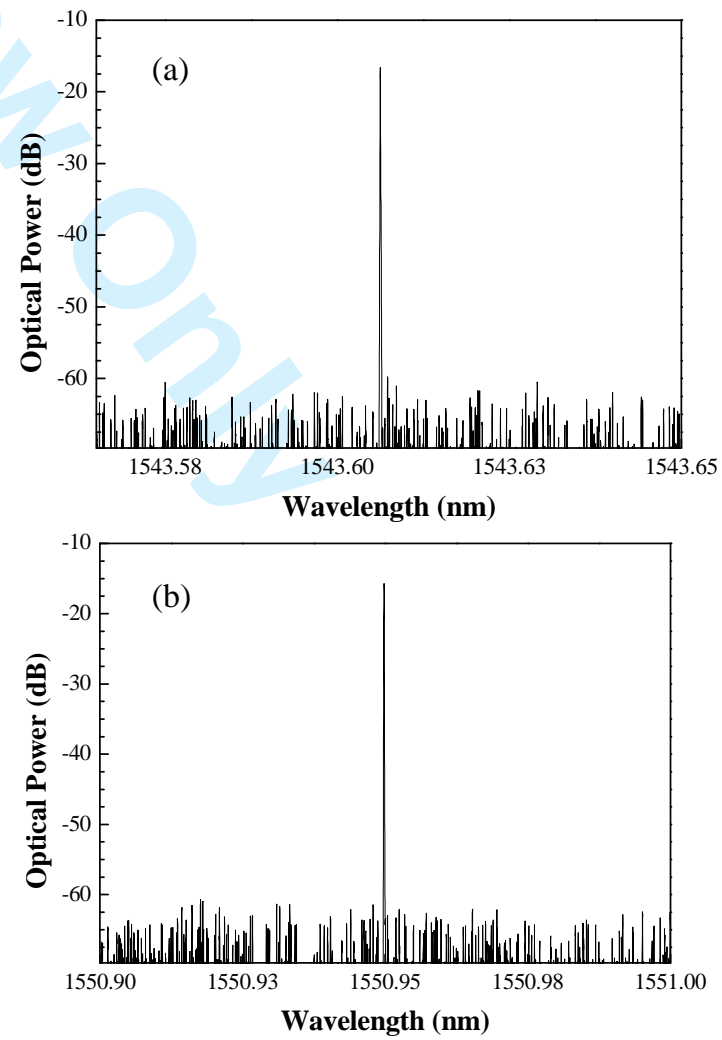

Fig. 4. Details of the output optical spectrum (a) for the first channel $\left(\lambda_{1}=\right.$ $1543.6 \mathrm{~nm})$ and (b) for the second channel $\left(\lambda_{1}=1550.9 \mathrm{~nm}\right)$ at dualwavelength operation. 